\title{
La «vía media» española del modelo de bienestar mediterráneo
}

\section{Luis Moreno}

Consejo Superior de Investigaciones Científicas

Lmorfer@iesam.csic.es

\section{Resumen}

El Estado de bienestar español incorpora elementos de las tradiciones «bismarckiana» y «beveridgeana», y cabe ser considerado como una «vía media» respecto a otros regímenes de protección social de las democracias avanzadas. Ha alcanzado, asimismo, un nivel intermedio de desmercantilización (de-commodification) y de acceso universal y por comprobación de medios (means testing) a prestaciones y servicios sociales. El artículo analiza a grandes rasgos las características del régimen mediterráneo del bienestar, y se concentra en un análisis puntual del desarrollo de los programas de asistencia social y servicios sociales en España. La lógica implícita en la federalización autonómica, así como el principio de subsidiariedad territorial de la Unión Europea, auspician una mayor participación de los niveles regionales y locales en la provisión de políticas sociales, los cuales pueden optimizar los criterios de eficiencia y exigencia democrática.

Palabras clave: régimen del bienestar mediterráneo, política social, servicios sociales, Estado de las Autonomías.

\section{Abstract. The Spanish «mid way» to the Mediterranean Welfare State Model}

The Spanish Welfare State incorporates elements of both Bismarckian and Beveridgean traditions, and can be labelled as a via media with respect to other systems of social protection in advanced democracies. It also represents a middle way of de-commodification and gendering, and of universal and means-tested access to services and benefits. This article analyses the main features of the Mediterranean welfare regime, and concentrates in the development of the programmes of social assistance and social services in Spain. The rationale implicit in the process of federalisation in Spain, and that of the territorial subsidiarity in the European Union, favours the participation of sub-state layers of government in the running of social policies according to criteria of efficiency and of democratic accountability.

Key words: Mediterranean welfare regime, social policy, social services, Spanish «State of Autonomies».

\section{Sumario}

\section{Introducción 4. Conclusión}

2. El régimen mediterráneo del bienestar

Bibliografía

3. Asistencia social y servicios sociales en la España autonómica 


\section{Introducción}

$\mathrm{El}$ «enfoque de los regímenes», propuesto por Gøsta Esping-Andersen (1990¹), ha sido el más influyente en el debate conceptual y metodológico sobre el welfare de los últimos años. Según éste enfoque, los estados del bienestar se caracterizan por una constelación de encajes institucionales de carácter económico, político y social. La tarea para el científico social es la de identificar normativamente aquellos elementos cuya interacción afecta al conjunto de la política social y al bienestar ciudadano. La tipología elaborada por el sociólogo danés se basa principalmente en el análisis de tres grandes dimensiones: las relaciones entre estado y mercado ${ }^{2}$, la estratificación ${ }^{3}$ y la desmercantilización ${ }^{4}$ (de-commodification).

Sucede, sin embargo, que el establecimiento de relaciones causales en el "enfoque de los regímenes» parte de una cierta premisa de continuidad y estaticidad $^{5}$, en detrimento de las explicaciones que afectan al cambio social y a las transformaciones institucionales. De este modo, se tiende a observar de manera estática los futuros desarrollos del Estado de bienestar de acuerdo con las lógicas internas de las tres categorías principales expuestas por EspingAndersen, a saber, anglosajona, continental y escandinava.

1. El título original del libro publicado por la editorial Polity Press en 1990 (The Three Worlds of Welfare Capitalism), hace referencia a las economías de mercado de las democracias occidentales, en términos de T.H. Marshall, es decir, respecto al capitalismo democrático del bienestar.

2. Una dimensión que, en un primer momento, no tomaba cabalmente en consideración las relaciones entre estado y familia. El propio Esping-Andersen ha reconocido posteriormente (1999) el carácter estructurante que los hogares poseen en la conformación de los regímenes del bienestar, y al cual no había prestado suficiente atención.

3. Que pretende medir el grado en que los subsidios públicos tienden a segmentar o a integrar a los ciudadanos. Es decir, hasta qué punto los efectos de las políticas sociales contribuyen a generar nuevas desigualdades entre los ciudadanos o, por el contrario, las aminoran.

4. Concepto que se alinea con las ideas de Karl Polanyi (1957) y Richard Titmuss (1981) al valorar a las políticas sociales como medios para no depender del salario como única forma de emancipación y satisfacción de necesidades. El concepto de «desmercantilización» (decommodification) ha sido empleado por Esping-Andersen como un instrumento analítico que concierne a aquellos derechos sociales del trabajador que le permitirían cubrir sus necesidades vitales al margen del mercado laboral. (Nótese que ello no implica conceptualmente el rechazo a considerar el trabajo como mercancía dentro del sistema productivo.) El nivel de «desmercantilización» de los sistemas asistenciales estaría determinado por: a) las restricciones institucionales establecidas en el acceso del trabajador al conjunto de los derechos sociales; $b$ ) sus condiciones de elegibilidad; $c$ ) la relación entre salarios y prestaciones, y d) la existencia o no de un subsidio social de carácter universal para todos los ciudadanos.

5. El propio Esping-Andersen (1999) reconoce que la primera exposición de su tipología estaba constreñida y referida a una condiciones socioeconómicas en las economías capitalistas avanzadas prevalentes en los años 70 y 80 , caracterizadas por: a) unos sistemas de producción masivos (fordistas); b) unas estructuras de clases en las que el trabajador, fundamentalmente varón, era el arquetipo de ciudadano sujeto de derechos y obligaciones, y c) unas sociedades en las que la gran mayoría de los hogares se estructuraban en torno a la presencia de un solo miembro familiar perceptor de salarios. 
Desarrollando el paradigma analítico de los «recursos del poder» (power resources approach $)^{6}$, Esping-Andersen ha teorizado sobre los tres mundos del bienestar capitalista asumiendo implícitamente planteamientos socialdemócratas de orientación estatalista (state-centered) a la "escandinava», según los cuales la acción del estado central es la gran variable independiente que determina la producción de bienestar. Según esta perspectiva, las estructuras estatales disponen de una relativa autonomía que las hace responsables principales del desarrollo de los sistemas nacionales de provisión social.

Además, se enfatizan los recursos de poder de los actores colectivos y, en particular, de las organizaciones de trabajadores en la formación y acción posterior de las coaliciones de poder, consideradas como responsables de la implantación de estructuras de protección social. Aún en menor medida, también se toma en cuenta el legado histórico de las instituciones estatales como elementos principales en la consolidación de los distintos regímenes de bienestar (path dependency). Una obvia crítica al institucionalismo estatalista de la perspectiva de los «recursos de poder» es que a menudo pasa por alto el simple hecho de que el Estado de bienestar "[...] ha hecho concordante con la modernidad muchas funciones previamente desarrolladas por la familia, la iglesia, el gremio y la comunidad local» (Flora y Heidenheimer, 1981: 6).

La experiencia del Estado de bienestar acotada en el espectro ideológico (socialdemocracia) y en las coordenadas de espacio y tiempo (norte de Europa, tras la Segunda Guerra Mundial), ha servido como principal ejemplo de contraste respecto al cual se han tipologizado otros sistemas de protección social. Los estudios de bienestar, en general, han tendido a ser "suecocentristas». Así, el Estado de bienestar escandinavo se ha constituido para muchos analistas en vara de medir los logros y carencias de los sistemas de protección social del resto de los países capitalistas avanzados.

Buena parte de los análisis normativos de mayor influencia académica no han ocultado sus preferencias por el desarrollo del Estado de bienestar en Suecia. Sucede, empero, que sus consideraciones prescriptivas adolecen a menudo de voluntarismo, ideologismo y, sobre todo, de falta de conmensurabilidad comparativa $^{7}$. Se ha producido con frecuencia, por tanto, una mixtificación en la evaluación normativa respecto a qué debe ser un Estado de bienestar con el desarrollo histórico y real de los sistemas de protección social a analizar (Baldwin 1992).

6. La escuela de los «recursos de poder» subraya el valor autónomo de la política como instrumento de cambio y cuestiona la idea de que la política social simplemente refleja las necesidades sistémicas de las sociedades capitalistas e industrializadas.

7. Al fin y al cabo, Suecia es un pequeño país de ocho millones de habitantes, étnica y socialmente homogéneo, con unas características específicas (como son su moderna tradición estatalista), y en donde la intervención pública ha gozado de una alta legitimidad ciudadana. 


\section{El régimen mediterráneo del bienestar}

En los últimos años se ha teorizado un modelo distintivo de la Europa del sur que incluye básicamente a España, Grecia, Italia y Portugal (Ferrera, 1995; Moreno, 1997b). Se ha debatido si este tipo de bienestar mediterráneo es la mera expresión de una "familia de naciones» (Castles, 1993), rezagadas respecto al modelo «continental corporatista» al que pertenecen (Katrougalos, 1996), o si esta periferia o "fleco latino» es mera expresión de un nivel rudimentario de provisión social y desarrollo institucional (Leibfried, 1992; Gough, 1996).

A fin de sistematizar el argumento de la existencia un Estado de bienestar de la Europa del sur, se requiere más investigación de carácter comparativo. No obstante, y con carácter general, puede contrastarse que los cuatro países meridionales comparten analogías respecto a su historia, sistemas de valores y peculiaridades institucionales. Todos ello han sufrido, en distinto grado y duración, experiencias de dictaduras y gobiernos autoritarios durante el siglo XX, y han experimentado «retrasos» en los procesos de modernización (excepto en las primeras regiones industrializadas de España e Italia) (Giner, 1995).

El factor religioso ha sido de una gran relevancia estructural en los cuatro países, aunque la actuación de la iglesia como principal proveedor de protección social ha disminuido. Este proceso ha ido parejo con un mayor grado de secularización de las prácticas sociales en la Europa del sur. Los efectos de los procesos de europeización y globalización han estimulado una mayor convergencia con los países de la Europa central y septentrional (Unión Económica y Monetaria).

La Europa del bienestar meridional puede ser calificada como una «vía media» u opción intermedia entre los regímenes «bismarckianos», de mantenimiento de rentas ocupacionales, y los «beveridgeanos», de cobertura universalística. En el conjunto de estados industrialmente avanzados de la OCDE, los países de la Europa del sur reflejan un nivel de valores medios respecto al grado de desmercantilización (de-commodification), a las relaciones entre los géneros, y al acceso universal a servicios y prestaciones de bienestar por comprobación de medios (means testing). Al analizar los incrementos de gasto social en porcentaje del PIB, se constata un mayor grado de convergencia de los países latinos con respecto al promedio de la Unión Europea (cuadro 1).

El caso de Italia se ajusta a un modelo "ocupacional mixto" que incorpora las características básicas del régimen contributivo continental, pero con algunos rasgos del régimen universalístico (Ferrera, 1993). Pero quizá sea el caso de España el que mejor representa dicha posición equidistante entre los procesos de universalización de sus sistemas de la salud, educativos y de pensiones, y la permanencia de una seguridad social de carácter contributivo. España es, pues, cabal representante de la "vía media» mediterránea (Moreno y Sarasa, 1992, 1993).

Aún dejando constancia de que existen particularidades entre los países meridionales, tanto en España como en Grecia, Italia y Portugal pueden obser- 
Cuadro 1. Gasto social en los países de la UE-12 (en porcentajes del PIB).

\begin{tabular}{lcccc}
\hline & $\mathbf{1 9 8 0}$ & $\mathbf{1 9 8 5}$ & $\mathbf{1 9 9 3}$ & $\mathbf{\Delta} \% \mathbf{( 1 9 8 0 - 9 3 )}$ \\
\hline Alemania & $\mathbf{2 8 , 8}$ & $\mathbf{2 8 , 4}$ & 31,0 & $+2,2$ \\
Bélgica & 28,0 & 29,3 & 27,6 & $-0,4$ \\
Dinamarca & 28,7 & 27,8 & 33,2 & $+4,5$ \\
Francia & 25,4 & 28,8 & 30,9 & $+5,5$ \\
Irlanda & 20,6 & 23,6 & 21,4 & $+0,8$ \\
Luxemburgo & 26,5 & 23,4 & 24,9 & $-1,6$ \\
Países Bajos & 30,1 & 31,7 & 33,6 & $+3,5$ \\
Reino Unido & 21,5 & 24,3 & 27,8 & $+6,3$ \\
\hline UE-12 & $\mathbf{2 4 , 3}$ & $\mathbf{2 6 , 0}$ & $\mathbf{2 7 , 8}$ & $+3,5$ \\
\hline España & 18,2 & 20,0 & 24,0 & $+5,8$ \\
Grecia & 9,7 & 15,4 & 16,3 & $+6,6$ \\
Italia & 19,4 & 22,6 & 25,8 & $+6,4$ \\
Portugal & 12,8 & 14,1 & 19,5 & $+6,7$ \\
\hline
\end{tabular}

Nota: gastos corrientes y a precios de mercado.

Fuente: Eurostat (1995: 16).

varse tendencias de carácter demográfico y condiciones socioeconómicas similares. Con carácter general, tres son los rasgos que cabe considerar como diferencialmente característicos del régimen del bienestar mediterráneo: a) necesidades y estilos de vida diferentes; $b$ ) microsolidaridad familiar, y $c$ ) conjunción entre universalismo y selectividad.

a) En lo que afecta a la dimensión cultural y axiológica del desarrollo de sus sistemas de bienestar, en la Europa del sur se denota una autopercepción de necesidades y estilos de vida diferentes. A destacar son sus prácticas de repartos intrafamiliares, de régimen de propiedad de viviendas, y la heterogeneidad de sus patrones de reproducción social.

Particularmente relevante es la prevalencia de los valores de inclusión familiar, así como la redistribución de ámbito familiar durante los ciclos vitales (donaciones entre los miembros familiares, procesos de emancipación, y proliferación de empresas y empleos de ámbito familiar). Las prácticas de la puesta en común de recursos (resource pooling) y de presupuestos flexibles (soft budgeting) ${ }^{8}$ implican que el hogar (a veces compuesto por la familiar nuclear, a

8. En combinación con el resource pooling, el soft budgeting implica ciertas prácticas contables en el seno de hogares y familias. Según tales comportamientos, los componentes de una unidad doméstica asumen que aunque gasten más de lo que ellos aportan individualmente, algún otro miembro cubrirá el «déficit contable» producido (Petmesidou, 1996). 
veces por la familia extensa o complementado por la parentela) se constituye en una institución central de referencia en la procura de bienestar para sus miembros.

La formas de reproducción social en los países de la Europa del sur muestran patrones diferenciados, tales como el que afecta al régimen de propiedad de viviendas. No cabe duda que la vivienda, aún no siendo considerada estrictamente como un ámbito de intervención directa de las políticas sociales, conforma la naturaleza de los regímenes del bienestar en los distintos países. Sus repercusiones en la calidad de vida de los ciudadanos y sus implicaciones en los procesos de acumulación patrimonial son importantes en el desarrollo del bienestar.

En el caso de la Europa meridional, la concentración de la titularidad de las viviendas en las personas mayores subraya el rol decisivo del hogar como institución de compensación entre sus distintos componentes generacionales (Castles y Ferrera, 1996). Italia y España son los países con las tasas de mayor polaridad por edades en la propiedad inmobiliaria para uso doméstico. Además, las políticas de vivienda en el sur de Europa incentivan la compra y no el alquiler de viviendas. Ello, unido a las tardías edades de emancipación de los jóvenes, características de los países meridionales europeos, indican unos mayores usos de los recursos comunes disponibles en el hogar en comparación con las pautas de «individualización» familiar de otros regímenes del bienestar. Los efectos que todo ello provoca en el desarrollo de las políticas sociales son determinantes.

El agregado del bienestar (welfare mix) y el nexo género/familia/trabajo en los países mediterráneos son complementarios. Sus prácticas a menudo se traducen en formas institucionales particularistas y en una baja eficiencia en la provisión de servicios de protección social. Así, las organizaciones subsidiadas por el sector público constituyen en sí mismas un freno a reformas comprensivas generalistas, como ha sido el caso de los modelos anglosajón y escandinavo. Pero la reconstrucción de los Estados de bienestar meridionales ha reflejado un ajuste de los tradicionales enfoques voluntaristas de las subculturas políticas de la izquierda, así como del paternalismo y la discrecionalidad de la derecha. Tales procesos están en cualquier caso condicionados por los trasfondos culturales y las prácticas civiles -inciviles en el caso de los intereses corporativistas - de sus fragmentados sistemas sociales.

Ciertamente, las preferencias y prácticas culturales de los países de la Europa del sur han estructurado sus sociedades civiles de un modo peculiar y, a menudo, reticular. Los ciudadanos se adscriben a grupos o redes sociales de influencia que a menudo llevan a cabo prácticas de patronazgo, clientelismo y predación grupal. Las prácticas particularistas de grupos y redes institucionales implican los usos y abusos del "efecto Mateo». Recuérdese que éste hace referencia a las desproporcionadas ventajas para aquellos ciudadanos con recursos de información y de acceso a los servicios públicos.

9. Este efecto se relaciona con la aserción del Evangelio, según San Mateo, de que: «[...] a cualquiera que tiene, se le dará, y tendrá más; pero al que no tiene, aún lo que tiene le será quitado» $(13: 12)$. 
En el caso de la distribución de subsidios de invalidez en el Mezzogiorno italiano, la intermediación de los partidos políticos se ha constituido tradicionalmente en una práctica institucional generalizada, a menudo en connivencia con el fraude encubierto. En Grecia, y en menor medida en España, el acceso a redes clientelares a menudo subsidiadas por los poderes públicos constituye un cauce importante para la apropiación de recursos y privilegios. Tales usos y prácticas no favorecen una percepción de la solidaridad como un activo común del conjunto de la ciudadanía. En la Europa meridional la solidaridad adopta un carácter micro y se observa fundamentalmente en el seno de la familia.

b) El Estado de bienestar meridional descansa en gran medida en el papel desempeñado por la familia en la procura de satisfacción vital (well-being) y bienestar (welfare) para sus miembros. Las importantes transferencias intrafamiliares que se producen en la Europa mediterránea son de índole tanto material como inmaterial. No todas pueden cuantificarse en cifras, aunque buena parte de los cuidados que las mujeres prestan a sus hijos, a sus padres, o su mayor dedicación al trabajo doméstico son activos fundamentales que permanecen ocultos en la contabilidad nacional de estos países.

Son precisamente las transferencias de índole no estrictamente material las que conforman culturas claramente distinguibles en el caso mediterráneo, y sitúan a la familia como principal foco de socialización de los ciudadanos ${ }^{10}$. Los niveles afectivos entre los miembros familiares conllevan no sólo transmisión de actitudes, conocimientos, percepciones y valores reflejados en los altos niveles de ayuda mutua en el seno de los hogares, sino de una frecuente priorización de lo microcolectivo respecto a los intereses meramente individuales de los componentes de los hogares. Todo ello conforma una visión social en la que las estrategias familiares prevalecen en las formas de intercambio social entre los ciudadanos. Es precisamente en el seno del microcosmos de solidaridad familiar donde el rol de la mujer ha sido - y continúa siendo- determinante en el desarrollo de las políticas de bienestar.

A lo largo de los últimos decenios se ha producido una correlación entre el aumento del nivel de educación formal de la población femenina y un mayor deseo de incorporación de las mujeres en el mercado laboral formal ${ }^{11}$. La cre-

10. Según los resultados de la investigación dirigida por Tomás Calvo, casi tres cuartas partes de los escolares españoles consideraban a la familia como la fuente de socialización principal y en "[...] donde se dicen las cosas más importantes para la orientación en la vida». En segundo lugar, pero a más de 50 puntos, figuraba la escuela (El País, 03.02.98).

11. En España, durante los dos últimos períodos de crecimiento del empleo (1964-74 y 1985-1990), la participación laboral de la mujer se incrementó respecto a la masculina. En 1969 había 32 trabajadoras por cada 100 trabajadores. Este ratio aumentó a 39/100, en 1974, a 41/100, en 1985, y a 46/100 en 1990 (Garrido, 1994). Además la participación laboral de la mujer es mayor en el sector público que en el privado. En 1964 había 17 funcionarias en España por cada 100 empleados públicos. La proporción había variado drásticamente en 1991: 70/100 en 1991. Más revelador de estos cambios es el hecho de que, en 1990 y en el grupo de edad de 25 y 29 años, la proporción de funcionarias era de 105 por cada 100 trabajadores públicos varones. 
ciente participación laboral de las mujeres junto a los nuevos obstáculos para la formación y expansión familiar, conllevan grandes incógnitas respecto al desarrollo futuro del régimen mediterráneo (Guillén, 1997).

Dificultades estructurales para la creación de familias, tales como el mayor desempleo de los grupos de edad jóvenes, la carestía de la vivienda o la inadecuación entre instrucción adquirida y oferta de empleos, no han hecho renunciar a las mujeres mediterráneas a sus deseos de maternidad. Las bajas tasas de natalidad de los países del sur (siendo las de España e Italia las menores en Europa) no implican que el número de madres haya declinado sensiblemente. En general, los hijos se procrean a edades más tardías. Además, y a diferencia de patrones pasados de familias numerosas, el número de hijos por mujer fértil ha disminuido ${ }^{12}$. Pero no se ha incrementado el de aquellas mujeres que han renunciado a la maternidad por completo, cuyas cifras son mayores en países del centro y norte de europeo. Así, aunque ha crecido el numero de mujeres mediterráneas sin hijos, su tasa se mantiene por debajo de los estándares europeos (Sardon, 1990; Santini, 1998).

La seguridad y estabilidad en el empleo son elementos cruciales para las mujeres trabajadoras mediterráneas. Es por ello que, en contraste con países del régimen continental (Alemania) y anglosajón (Reino Unido), las interrupciones laborales de las mujeres en España - y en el conjunto de la Europa del sur - están motivadas en mayor medida por el nivel de educación y los intereses profesionales que por razones de maternidad. Ello se traduce en altas tasas de continuidad laboral y menor número de trabajos a tiempo parcial (Jurado y Naldini, 1996).

En el caso de las familias de la Europa del sur, la duplicidad de funciones dentro y fuera del hogar ha generado una nueva tipología de "supermujer» (superwoman) que ha actuado como un sólido amortiguador de sus sistemas de protección social. Merced a esta situación de hiperactividad femenina ${ }^{13}$, cabe afirmar que el régimen del bienestar mediterráneo ha podido solventar mejor los efectos producidos por los procesos de posindustrialización, globalización y reestructuración socioeconómica del último decenio del siglo XX en Europa. El papel cambiante de la mujer mediterránea respecto a sus expectativas vitales refleja, no obstante, actitudes de un "familismo ambivalente» (Flaquer, 1995, 1998; Saraceno, 1995). La evolución de su rol tradicional como principal inductora de la fuerte microsolidaridad familiar en la Europa del sur aparece como la variable independiente en todos y cada uno de los análisis prospectivos del futuro desarrollo del bienestar en la Europa del sur.

12. En el caso de Italia, por ejemplo, pueden distinguirse dos modelos reproductivos: en el centro y norte del país transalpino se ha consolidado en los últimos tiempos la familia con un hijo único, mientras en el sur el promedio se acerca al de 2 hijos (Saraceno, 1998; Naldini, 1999).

13. En Italia, la mitad de las mujeres ocupadas y con hijos trabajan un total de 60 horas a la semana. Un tercio de ellas trabajan más de 70 horas. Sólo el 15 por ciento de padres empleados con hijos trabajan 60 horas semanales. Nótese que la ausencia del marido en la casa se traduce en 2 horas menos de trabajo al día para las esposas (La Repubblica, 09.02.99). 
c) La conjunción entre universalismo y selectividad de las políticas del bienestar mediterráneas viene condicionada en gran medida por la naturaleza de sus mercados de trabajo. Si bien sus sistemas de seguridad social comparten con los de la Europa continental su filosofía contributiva, la intervención de sus gobiernos en los últimos años se ha dirigido a procurar un acceso universal a programas de salud, pensiones y servicios sociales a aquellos grupos al margen de la economía formal generadora de derechos asegurativos. En la Europa del sur dos características de sus mercados laborales son peculiares respecto a los otros regímenes del bienestar: la heterogeneidad de su fuerza laboral y el peso de la economía «sumergida».

En la Europa meridional existe una fractura en sus mercados de trabajo entre los altos niveles de protección de los ciudadanos formalmente empleados (trabajadores «fijos» y funcionarios), aquéllos en la periferia laboral (empleados en puestos "móviles», de entrada y salida en la zona asegurativa) y marginales (precarios, y con trabajos «basura» o en la "economía sumergida», que no generan derechos contributivos de la seguridad social).

Recuérdese también que la dimensión de las economías informales de la Europa del sur es muy considerable (existen estimaciones que la sitúan entre un 15 y un 25 por ciento del Producto Interior Bruto ${ }^{14}$. Ello comporta una desigual distribución de las cargas financieras entre los diversos grupos ocupacionales. Los cotizantes y contribuyentes cumplidores con sus deberes ciudadanos y de rentas más bajas son penalizados y viceversa. Dicha realidad dificulta la intervención pública a la hora de seleccionar políticas dirigidas a grupos (targeting), circunstancia que trata de solventar el acceso a la universalización de servicios y prestaciones (aunque mediante comprobación de medios de los beneficiarios en la mayoría de los casos).

Las reformas de las políticas del bienestar de clara vocación universalística se han generalizado en la Europa del sur. En España, por ejemplo, tras la puesta en vigor de la Ley de pensiones no contributivas de 1990, la cobertura para las pensiones de vejez (más de 65 años) y de invalidez (de más del 65 por ciento para los ciudadanos entre 18 y 65 años) pasó a ser universal ${ }^{15}$. La Ley General de la Sanidad de 1986 estableció el acceso universal a la salud de todos los españoles y residentes extranjeros en España. En el resto de los países mediterráneos es también en el área de la salud donde los criterios universalísticos se han hecho más palpables. En Portugal, el sistema nacional de salud fue instituido en 1979. Un año antes se produjo en Italia una reforma que fue prelu-

14. En el caso de Grecia, el porcentaje de la "economía negra» podría alcanzar la cifra del 45\% del Producto Interior Bruto (Eurostat, 1995).

15. Los requisitos generales son: a) residencia en España durante 5 años, con 2 ininterrumpidos con carácter previo a la percepción de la pensión, y b) carencia de recursos económi$\cos$ (evaluada mediante comprobación de medios del beneficiario). Nótese que en el período 1980-92 el número de pensionistas aumentó en 2,5 millones (2,1, contributivas, y 0,4, no contributivas), pasando de 4,8 a 7,2 millones. El gasto social por pensiones se incrementó del 5,9 por ciento del PIB (5,8 por ciento, contributivas, y 0,1 por ciento, no contributivas) al 8,6 por ciento $(8,1$, contributivas, y 0,5 , no contributivas). 
dio de la calificada como «reforma de las reformas» en 1992. Los múltiples y diversos programas ocupacionales de la salud fueron articulados en el Servizio Sanitario Nazionale. En Grecia el servicio nacional de la salud se constituyó por ley en $1983^{16}$.

Al afrontar las situaciones de pobreza y exclusión social, la mayor participación de los niveles de gobiernos regional y local en el desarrollo de redes de servicios sociales de base y de los programas de asistencia social está conformando una pauta institucional característica en la Europa del sur. En paralelo a la combinación de los principios de selectividad y universalismo, se observa por tanto un mayor empeño institucional por la descentralización. En Italia «la reforma de las reformas» del servicio sanitario nacional ha conllevado una descentralización de funciones organizativas y de gestión ${ }^{17}$. Además, la aprobación en fase experimental 1998 del programa nacional de rentas mínimas, reddito minimo di inserimento, ha previsto la descentralización de su gestión a las comunidades locales ${ }^{18}$. En Grecia y Portugal la descentralización de servicios, en su vertiente de gestión administrativa, ha sido más tenue ${ }^{19}$. Por contra, en España destaca el protagonismo de las comunidades autónomas en el establecimiento de sistemas regionales de servicios sociales y de asistencia

16. Sin embargo, los problemas de gestión han incidido en el mantenimiento de la diversidad ocupacional de los programas de atención sanitaria y, por tanto, han contribuido a la reproducción de pasadas desigualdades organizativas y cobertura (Petmesidou, 1996).

17. Las «unidades sanitarias locales» que conforman la estructura básica del Servizio Sanitario Nazionale han pasado a ser "empresas públicas» con una amplia autonomía organizativa. Sus gerentes son contratados por las regiones por periodos prorrogables de 5 por las regiones de acuerdo a criterios profesionales. El gobierno central mantiene competencias generales de planeamiento, financiando un conjunto estandarizado de servicios que garantice un nivel equivalente de prestación a todos los ciudadanos, sea cual fuere su región de residencia. Las regiones pueden suplementar mediante recursos propios (co-financiación de los usuarios o impuestos) los gastos de funcionamiento que establezcan las «unidades sanitarias locales». De igual manera, los superávits generados pueden destinarse a inversiones o al pago de incentivos al personal médico y administrativo (Ferrera, 1999),

18. El programa se ha implantado en 42 comunidades locales de forma experimental, con una sobrerrepresentación de aquéllas radicadas en el sur italiano donde la pobreza se halla más extendida. Las características son parecidas a las del $R M I$ francés y las rentas mínimas autonómicas españolas. Los beneficiarios reciben una prestación de unas 45.000 pesetas (o la diferencia hasta alcanzar esta cifra si disponen de medios propios) y deben realizar tareas de inserción (asistencias a cursos de formación o actividades socialmente útiles). Este programa será evaluado en el año 2000. Se prevé que la financiación se realizará con cargo al presupuesto nacional en un $90 \%$, siendo el $10 \%$ restante a cargo de las comunidades locales (Ferrera, 1999).

19. La escasa descentralización, tanto territorial como funcional, de servicios sociales, así como la reducida iniciativa de las asociaciones voluntarias, han subrayado el papel central de la acción del Estado central y del paraestatalismo de la Iglesia ortodoxa en la configuración del Estado de bienestar griego. A su vez, el referéndum del 8 de noviembre de 1998 en Portugal evidenció la negativa de los portugueses a la regionalización del estado lusitano. Recuérdese, no obstante, que la implantación de las regiones autónomas está reconocida en la Constitución de 1976, por lo que un mayor grado de descentralización y desconcentración no está descartada de cara al futuro. 
social $^{20}$, y en la implantación de los programas de rentas mínimas de carácter subestatal. En realidad, han sido las propias comunidades autónomas las que han apurado el «cierre» del sistema asistencial con el establecimiento de una «red mínima de seguridad» (Serrano y Arriba, 1998).

\section{Asistencia social y servicios sociales en la España autonómica}

La Constitución de 1978 marcó el inicio del proceso de institucionalización de los servicios sociales en la España democrática. De acuerdo con el artículo $148 ; 1.20$, la asistencia social es una competencia exclusiva de las comunidades autónomas. Los poderes relativos a la legislación básica y el régimen económico del sistema de seguridad social quedaron atribuidos al estado central. No obstante a ello, nacionalidades y regiones también podían ejercer competencias ejecutivas en la gestión de aquellos programas contributivos de la seguridad social que pudieran ser transferidos a ellas (art. 149; 1.17). Recuérdese que el texto constitucional ni definió ni delimitó específicamente ambas áreas de actuación (asistencia social y servicios sociales). Además todas aquellas competencias no relacionadas en la lista de "exclusiva» del estado central podían ser requeridas por las Comunidades Autónomas (art. 149.3).

Dado el carácter flexible de las disposiciones constitucionales, las comunidades autónomas reclamaron en sus estatutos de autonomía un considerable número de servicios y funciones en las áreas de asistencia social, servicios sociales, desarrollo comunitario y atención primaria, promoción social y, en general, políticas del bienestar. Los únicos servicios que las CC.AA. no solicitaron fueron los correspondientes al Instituto Nacional de Servicios Sociales (INSER$\mathrm{SO}$ ). Sin embargo, durante el decenio de los años noventa, las competencias ejecutivas para gestionar prácticamente todos los servicios sociales del sistema contributivo fueron transferidos a las comunidades autónomas.

Durante el período 1982-1993, mediante iniciativas legislativas autonómicas, se establecieron los sistemas regionales de servicios sociales en nacionalidades y regiones. Tales leyes autonómicas interpretaron extensivamente el concepto de asistencia social. Asumían implícitamente que los servicios sociales también eran una competencia "exclusiva» de las comunidades autónomas. Consiguientemente, todas las leyes autonómicas incorporaron el criterio de unos servicios públicos integrales del bienestar, con acceso abierto y sin discriminación para los ciudadanos de sus respectivas comunidades. Se procuró desarrollar la idea de «agregado del bienestar», incentivando la participación en la producción subsidiaria de servicios sociales por parte de las organizaciones no lucrativas, las cuales han sido subsidiadas en su mayor parte por los poderes públicos autonómicos (Sarasa, 1997).

20. Téngase en cuenta que buena parte de la reacción ciudadana a la burocratización mecanicista de la provisión centralizada, característica del anterior régimen franquista, ha tenido también su expresión en el deseo de protagonismo institucional de comunidades autónomas y municipios. 
En los años 1983-84 el gobierno del PSOE intentó infructuosamente introducir una ley nacional de servicios sociales que integrase los servicios dispersos en el sistema contributivo de la seguridad social en un marco institucional único y de gestión centralizada. En 1986 el Tribunal Constitucional dictaminó que, a pesar de las «competencias exclusivas» de nacionalidades y regiones, la administración central podría desarrollar programas de asistencia social garantizando un trato equitativo entre las comunidades autónomas (sentencia 146/86). Sin embargo, los mesogobiernos españoles se han consolidado como protagonistas institucionales del desarrollo del bienestar en España, consecuencia de la lógica constitucional por la descentralización de la asistencia social (Casado y otros, 1994).

En 1987, un importante acuerdo entre los tres niveles de la Administración pública se plasmó en la aprobación del Plan Concertado para el Desarrollo de Prestaciones Básicas de Servicios Sociales de las Corporaciones Locales ${ }^{21}$. El plan posibilitaba la cooperación intergubernamental con el objetivo de desarrollar servicios de atención primaria ${ }^{22}$.

En el año 1988 comenzó el ciclo expansivo de los servicios sociales en España, con un incremento generalizado del gasto público del bienestar. Se creó entonces el Ministerio de Asuntos Sociales, que llevó a cabo una labor de coordinación con los mesogobiernos autonómicos para el desarrollo de los planes generales, tales como los relativos a la vejez, drogadicción, igualdad de oportunidad o juventud. No se trataba de leyes o normas de desarrollo legislativo, pero su funcionalidad estribó en allanar el camino para la futura transferencia del INSERSO a los sistemas regionales de servicios sociales.

Además del desarrollo de las redes autonómicas de servicios sociales, con la asunción de tareas ejecutivas y de gestión de los servicios sociales otrora dependiente de la seguridad social, las comunidades autónomas han implantado nuevas políticas sociales de carácter asistencial. A la miscelánea de subsidios heredados de los tradicionales programas de beneficencia pública, y a administración de las pensiones no contributivas, las nacionalidades y regiones han contribuido con políticas innovadoras, como son los programas de rentas mínimas de inserción (Aguilar, Gaviria y Laparra, 1995).

Otras prestaciones de tipo asistemático y contingencial son provistas, principalmente a nivel municipal, a ciudadanos en estado de inmediata necesidad. Todo ello ha conformado una «red de seguridad» en España de composición fragmentada pero comprensiva y en vías de consolidación (Rodríguez Cabrero,

21. El Plan no fue suscrito por la Comunidad Autónoma Vasca, dado que no aceptó financiamiento condicionado o categórico para el desarrollo de la red de centros de atención primaria en Euskadi. Recuérdese que el financiamiento anual del Plan se realiza a partes iguales con cargos presupuestarios a los niveles local, regional y central implicados.

22. Los servicios municipales a desarrollar abarcaban las áreas de: a) información y asesoramiento; $b$ ) servicios sociales de día para discapacitados y mayores; $c$ ) centro de acogida para mujeres maltratadas, madres solteras, huérfanos o menores maltratados y centro para transeúntes, y d) prevención e integración social. 
1994). La gran expansión de asistencia social y servicios sociales se manifiesta en una dualización de iure entre trabajadores con empleos estables (insiders) y aquellos otros ciudadanos excluidos del mercado laboral formal (outsiders). Empero ambas dimensiones están interrelacionadas de facto, formando un agregado público de provisión del bienestar (Moreno y Arriba, 1999).

\section{Conclusión}

España, un Estado de composición plural, asiste en la última parte del siglo XX a la federalización de su organización política territorial (Moreno, 1997a). Desde 1978 hasta 1995, la descentralización competencial del Estado de las Autonomías se ha traducido en una modificación sustancial de la participación de los tres niveles gubernamentales en el gasto público general (cuadro 2). En los escenarios futuros del desarrollo del bienestar en España, el papel a desempeñar por las comunidades autónomas y los municipios será, por tanto, fundamental.

Los programas y servicios ex novo puestos en vigor al nivel autonómico y local han estimulado el "principio del agravio comparativo» y la «regla de la mimesis autonómica» en una espiral de incentivación y de renovación de los servicios sociales. El carácter contractual «abierto» de los ajustes políticos entre los tres niveles de la Administración pública española aparece como la principal variable delimitadora de las formas que el Estado de bienestar adoptará en el futuro en España.

La federalización en curso en España se inscribe plenamente con el principio de subsidiariedad que conforma el vigente proceso de convergencia europea. Sin embargo, las responsabilidades en la actuación de los municipios aún restan por encajarse. Se denota, en este sentido, un creciente protagonismo recentralizador de los niveles intermedios de gobierno. Es ésta una tendencia que cuestiona las demandas por la «reinvención» de las instituciones municipales, las cuales se reclaman como garantes principales de la eficiencia en la provisión de los servicios sociales de base.

A menudo sucede, como es el caso español, que la transferencia de responsabilidades y competencias a las administraciones locales no va acompañada de las adecuadas dotaciones presupuestarias. Los trabajadores sociales de

Cuadro 2. Distribución territorial del gasto público en España (\%)

\begin{tabular}{lrrrrrrc}
\hline & $\mathbf{1 9 8 1 ^ { 1 }}$ & $\mathbf{1 9 8 4}$ & $\mathbf{1 9 8 7}$ & $\mathbf{1 9 9 0}$ & $\mathbf{1 9 9 2}$ & $\mathbf{1 9 9 7}$ & $\mathbf{1 9 9 9}^{\mathbf{2}}$ \\
\hline Central & 87,3 & $\mathbf{7 5 , 6}$ & $\mathbf{7 2 , 6}$ & $\mathbf{6 6 , 2}$ & $\mathbf{6 3 , 0}$ & 59,5 & 54 \\
Autonómico & 3,0 & 12,2 & 14,6 & 20,5 & 23,2 & 26,9 & 33 \\
Local & 9,7 & 12,1 & 12,8 & 13,3 & 13,8 & 13,6 & 13 \\
\hline
\end{tabular}

1. Generalización del proceso autonómico.

2. Estimaciones gubernamentales.

Fuente: Ministerio de Administraciones Públicas (MAP, 1997). 
base se encuentran, así, en el rompeolas de unas actuaciones mediatizadas por unos objetivos a menudo contradictorios. Se les requiere, por ejemplo, conocer y analizar detalladamente los casos que se les presentan, pero al mismo tiempo se ven impelidos a atender el mayor número posible de usuarios. Deben ser, al mismo tiempo, negociadores y ejecutores de programas en muchos casos infradotados y de poca visibilidad para el conjunto ciudadano. Además, la actuación complementaria de las asociaciones del tercer sector depende en muchos casos de los recursos públicos transferidos localmente. En dicha situación, la sociedad civil local corre el riesgo de pasar de ser subsidiaria a estar subsidiada, y los trabajadores sociales deben desplegar una capacidad doble de coordinación y supervisión.

En conclusión, el proceso de federalización autonómica en España confronta un desarrollo futuro en el que los niveles subestatales de la intervención pública aparecen en condiciones de poder optimizar ambos criterios de eficiencia y exigencia democrática requeridos con carácter general en la provisión de las políticas públicas. Además, tal proceso descentralizador se alinea con el principio de subsidiariedad imperante en el proceso de europeización. De los niveles de integración social alcanzados en los niveles más próximos al ciudadano dependerá en gran parte la renovación del pacto social por la legitimación del Estado de bienestar en España en el umbral del tercer milenio.

\section{Bibliografía}

Aguilar, M.; GaViria, M.; LAPARRA, M. (1995). La caña y el pez. El salario social en las comunidades autónomas 1989-1994. Madrid: Fundación FOESSA.

BALDWIN, P. (1992). «The Welfare State for Historians. A Review Article». Comparative Studies in Society and History, 34 (4), p. 695-707.

CASADO, D. y otros (1994). «Acción social y servicios sociales». FOESSA, V Informe Sociológico sobre la situación en España. Sociedad para todos en el año 2000. Madrid: Fundación FOESSA, p. 1735-1880.

Castles, F. (1993). Family of Nations. Patterns of Public Policy. Nueva York: Oxford University Press.

CAstles, F; Ferrera, M. (1996). «Home Ownership and the Welfare State: Is Southern Europe Different?». South European Society \& Politics, 1 (2), p. 163-185.

CRUZ RoCHE, I. (1994). «La dinámica y estructura de la universalización de las pensiones». V Informe Sociológico sobre la Situación Social en España. Madrid: Fundación FOESSA, p. 1495-1505.

Esping-ANDERsen, G. (1990). Three Worlds of Welfare Capitalism. Cambridge: Polity Press. Ed. castellana (1993): Los tres mundos del Estado de bienestar. Valencia: Edicions Alfons el Magnànim.

- (1999). Social Foundations of Postindustrial Economies. Oxford: Oxford University Press.

Eurostat (1995). Eurostat Yearbook 1995: A Statistical Eye on Europe, 1980-93. Luxemburgo: OOPEC.

FERRERA, M. (1993). Modelli di solidarieta: Politica e riforme sociali nelle democrazie. Bolonia: Il Mulino. 
- (1995). «Los Estados de bienestar del Sur en la Europa social». En SARASA, S.; Moreno, L. (ed.). El Estado de bienestar en la Europa del sur. Madrid: CSIC, p. 85-111.

- (1999). "Targeting Welfare in a "Soft" State». En GilberT, N. (ed.). Targeting Social Benefits. New Brunswick, N.J.: Transaction Books (en prensa).

FLAQUER, L. (1995). «El modelo de familia española en el contexto europeo». En SARASA, S.; MORENO, L. (ed.). El Estado de bienestar en la Europa del sur. Madrid: CSIC, p. 261-288.

FlorA, P.; HeIDENHEIMER, A.J. (1981). «The Historical Core and Changing Boundaries of Welfare States». En Flora, P.; Heidenheimer, A.J. (ed.). The Development of Welfare States in Europe and America. New Brunswick, N.J.: Transaction Books, p. 17-34.

GARRIDO, L. (1994). «La evolución de la situación de la mujer». VInforme Sociológico sobre la Situación Social en España. Madrid: Fundación FOESSA, p. 1315-1327. GINER, S. (1995). «La modernización de la Europa meridional». En SARASA, S.; MORENO, L. (ed.). El Estado de bienestar en la Europa del sur. Madrid: CSIC, p. $9-59$.

Gough, I. (1996). «Social Assistance in Southern Europe». South European Society \& Politics, 1 (1), p. 1-23.

GuilléN, A.M. (1997). «Regímenes de bienestar y roles familiares: un análisis del caso español». Papers, 53, p. 45-63.

Jurado, T.; NALDini, M. (1996). Is the South so different? Italian and Spanish Families in Comparative perspective. Working Paper 1/12. Mannheim Centre for European Social Research.

Katrougalos, G.S. (1996). «The South European Welfare Model: The Greek Welfare State in Search of an Identity». Journal of European Social Policy, 6 (1), p. 40-60. Leibfried, S. (1992). «Towards a European Welfare State? On integrating Poverty Regimes into the European Community». En FERGE, Z.; KolBerG, J. (ed.). Social Policy in a Changing Europe. Boulder, CO: Westview, p. 245-279.

LynCH, J. (1999). "Age-Bias in Social Policy in OECD Countries». Seminar Paper WS/28. Florencia: IUE/European Forum.

MAP (1997). Estudio sobre reparto del gasto público en 1997 entre los distintos niveles de administración. Madrid: Ministerio de Administraciones Públicas.

Moreno, L. (1997a). La federalización de España. Poder político y territorio. Madrid: Siglo XXI.

- (1997b). The Spanish development of Southern Welfare. Madrid: IESA (serie Documentos de Trabajo, 97-04). (http://www.csic.es/iesa).

Moreno, L.; Arriba, A. (1999). Welfare and Decentralization in Spain. Florencia: EUI (European Forum, WP 99/8).

Moreno, L.; Sarasa, S. (1992). The Spanish "Via Media» to the Development of the Welfare State. Working Paper 92-13. Madrid: Instituto de Estudios Sociales Avanzados (IESA).

- (1993). "Génesis y desarrollo del Estado de bienestar en España». Revista Internacional de Sociologia, 6 (diciembre), p. 27-69.

NALDINI, M. (1999). Evolution of Social Policy and the Institutional Definition of Family Models. The Italian and Spanish Cases in a Historical and Comparative Perspective. Tesis doctoral. Florencia: Instituto Universitario Europeo.

Petmesidou, M. (1996). «Social Protection in Southern Europe: Trends and Prospects». Journal of Area Studies, 9, p. 95-125. 
Polanyi, K. (1957). The Great Transformation: The Political and Economic Origins of Our Time. Boston: Beacon Paperback. Ed. castellana (1989): La gran transformación. Crítica del liberalismo económico. Madrid: Ediciones La Piqueta.

Rodríguez Cabrero, G. (1997). «Por un nuevo contrato social: el desarrollo de la reforma social en el ámbito de la Unión Europea». En Moreno, L. (ed.). Unión Europea y Estado de bienestar. Madrid: CSIC, p. 3-30.

Santini, A. (1998). «La fecondità». En Barbagli, M.; Saraceno, C. (ed.). Lo Stato delle famiglie in Italia. Bolonia: Il Mulino, p. 113-121.

SARACENO, C. (1995). «Familismo ambivalente y clientelismo categórico en el Estado de bienestar italiano». En SARASA, S.; MORENO, L. (ed.). El Estado de bienestar en la Europa del sur. Madrid: CSIC, p. 261-288.

- (1998). Mutamenti della famiglia e politiche sociali in Italia. Bolonia: Il Mulino.

SARASA, S. (1997). «El Tercer Sector en transición». En MorenO, L. (ed.). Unión Europea y Estado de bienestar. Madrid: CSIC, p. 345-372.

SARDON, J.P. (1990). Cohort fertility in member states of the Council of Europe. Population Studies, 21. Estrasburgo: Consejo de Europa.

Serrano, A.; Arriba, A. (1998). ¿Pobres o excluidos? El Programa del Ingreso Madrileño de Integración en perspectiva comparada. Madrid: Fundación Argentaria-Ministerio de Trabajo y Asuntos Sociales-Editorial Visor.

TrTmuss, R. (1981). Política social. Madrid: Ariel. Ed. original (1974): ABEL-SMiTH, B.; Titmuss, K. (ed.). Social Policy. An introduction. Londres: George Allen \& Unwin. 